toxicity $\geq \mathrm{G} 2$ was observed in 111 (42.7\%) pts, 65 (25\%) pts presented with infections and 44 (17\%) pts with febrile neutropenia.

Bleomycin lung-toxicity (BLT) was documented in 25 (18\%) pts: 6 (4\%) pts had mild toxicity (radiologic changes only), 12 (9\%) pts had severe toxicity (leading to hospitalization), in 7 (5\%) pts severity was unknown.

In 58 (24\%) pts treatment was discontinued prematurely due to toxicity. 171 (68.7\%) pts achieved complete remission, 30 (12\%) partial response. 58 (23\%) pts relapsed/progressed after first line treatment. $25(23 \%)$ pts died of $\mathrm{HL}$ and $15(14 \%)$ of treatment toxicity. With a median follow-up of 4.1 years (range: 0.05 - 17.95) for the whole study population, progression-free survival (PFS) at 2 and 5 years was $81 \%$ and $72 \%$ respectively, cause-specific survival (CSS) at 2 and 5 years was $85 \%$ and $78 \%$, respectively. CSS for pts $71-80$ years vs $60-70$ years; $H R=3.25, p<0.001$ and $>80$ years vs $60-70$ years; $H R$ $3.84, \mathrm{p}=0.001$.

Conclusion: Cause-specific survival of unselected, elderly $\mathrm{HL}$ pts > 71 years decreased significantly in comparison to those 60 to 70 years. Toxicities appeared to be relevant, in particular infections and BLT. Bleomycin needs to be used with extreme caution in this particular group of patients. New treatment strategies with a low toxicity profile are clearly needed, in particular for frail pts and pts older than 70 years.

Keywords: elderly; Hodgkin lymphoma (HL).

Disclosures: Moccia, A: Other Remuneration: Advisory Roche, Janssen, Takeda.

\section{5 \\ EXPLORATORY BIOMARKER ANALYSIS IN THE PH 3 ECHELON-1 STUDY: WORSE OUTCOME WITH ABVD IN PATIENTS WITH ELEVATED BASELINE LEVELS OF SCD30 AND TARC}

\author{
$\underline{\text { J. Radford }}^{1}$ | J.M.Connors ${ }^{2}$ | A. Younes ${ }^{3}$ | \\ A. Gallamini $^{4}$ | S.M. Ansell ${ }^{5}$ | W.S. Kim ${ }^{6}$ | \\ J. Cheong ${ }^{7}$ | I. Flinn ${ }^{8}$ | N. Kalakonda ${ }^{9}$ | \\ M. Kaminski $^{10}$ | R. Pettengell ${ }^{11}$ | M. Onsum ${ }^{12}$ | \\ N. Josephson ${ }^{13}$ | S. Kuroda ${ }^{14}$ | R. Liu ${ }^{15}$ | \\ H. Miao ${ }^{16}$ | A. Gautam ${ }^{17}$ | W.L. Trepicchio ${ }^{18}$ | \\ A. Sureda ${ }^{19}$
}

${ }^{1}$ Department of Medical Oncology, University of Manchester and the Christie NHS Foundation Trust, Manchester Academic Health Science Centre, Manchester, United Kingdom; ${ }^{2}$ Centre for Lymphoid Cancer, British Columbia Cancer Centre for Lymphoid Cancer, Vancouver, Canada; ${ }^{3}$ Division of Hematologic Oncology, Memorial Sloan Kettering Cancer Center, New York, United States; ${ }^{4}$ Research, Innovation and Statistics Department, A Lacassagne Cancer Centre, Nice, France; ${ }^{5}$ Department of Medicine, Mayo Clinic, Rochester, United States; ${ }^{6}$ Hematology-Oncology, Samsung Medical Center, Seoul, Republic of Korea; ${ }^{7}$ Division of Hematology, Department of Internal Medicine,
Yonsei University College of Medicine, Seoul, Republic of Korea; ${ }^{8}$ Department of Oncology, Sarah Cannon Research Institute, Nashville, United States; ${ }^{9}$ Molecular and Clinical Cancer Medicine, University of Liverpool, Liverpool, United Kingdom; ${ }^{10}$ Internal Medicine, University of Michigan, Ann Arbor, United States; ${ }^{11}$ Haematology, St George's Hospital, London, United Kingdom; ${ }^{12}$ Biomarkers, Seattle Genetics, Inc., Bothell, United States; ${ }^{13}$ Clinical Development, Seattle Genetics, Inc., Bothell, United States; ${ }^{14}$ Biostatistics, Takeda Development Center Japan, Takeda Pharmaceutical Company Limited, Osaka, Japan; ${ }^{15}$ Biostatistics, Millennium Pharmaceuticals Inc., a wholly owned subsidiary of Takeda Pharmaceutical Company Limited, Cambridge, United States; ${ }^{16}$ OTAU Clinical Research, Millennium Pharmaceuticals Inc., a wholly owned subsidiary of Takeda Pharmaceutical Company Limited, Cambridge, United States; ${ }^{17}$ Global Medical Affairs, Millennium Pharmaceuticals Inc., a wholly owned subsidiary of Takeda Pharmaceutical Company Limited, Cambridge, United States; ${ }^{18}$ Translational and Biomarker Research, Millennium Pharmaceuticals Inc., a wholly owned subsidiary of Takeda Pharmaceutical Company Limited, Cambridge, United States; ${ }^{19}$ Clinical Hematology, Institut Català d'Oncologia - Hospital Duran i Reynals, Barcelona, Spain

Introduction: Soluble (s)CD30 and thymus and activation-regulated chemokine (TARC) are established prognostic biomarkers in Hodgkin lymphoma $(\mathrm{HL})$ : higher baseline serum levels are associated with poorer survival outcomes. Elevated SCD30 and TARC levels are also associated with established poor prognostic factors in HL, e.g. Stage IV disease, higher International Prognostic Score (IPS), and extranodal involvement (ENI). The phase 3 ECHELON-1 study compared frontline brentuximab vedotin (a CD30-directed antibody-drug conjugate) plus doxorubicin, vinblastine, and dacarbazine (A+AVD) vs ABVD in patients (pts) with advanced classical $\mathrm{HL}$ (cHL). A+AVD demonstrated superior modified progression-free survival (modified PFS) vs ABVD $(\mathrm{HR}=0.77[95 \% \mathrm{Cl} 0.60-0.98] ; \mathrm{p}=0.035 ; 2-\mathrm{yr} \mathrm{mPFS} 82.1 \%$ vs 77.2\%. An exploratory ad-hoc biomarker analysis evaluated mPFS according to baseline sCD30 and TARC levels.

Methods: Serum samples were collected from 1334 pts with Stage III (36\%) or IV (64\%) $\mathrm{cHL}$ during the screening period and analyzed using validated assays for sCD30 (Covance Labs) and TARC (ICON Labs). mPFS (defined as time to progression, death, or evidence of noncomplete response followed by subsequent anticancer therapy) per independent review facility (IRF) was analyzed according to baseline SCD30 and TARC levels; the association of biomarker levels with treatment outcomes along with other potential predictive factors was explored in a multivariate Cox model.

Results: For the ad-hoc sCD30 analysis, pts were dichotomized around the median sCD30 baseline level $(207.9 \mathrm{ng} / \mathrm{mL})$. Pts in the A +AVD arm performed similarly regardless of baseline sCD30 level, with a 2-yr mPFS of $80.7 \%$ (sCD30 >median) and $82.7 \%$ (sCD30 $\leq$ median). However, a decrease in effectiveness of ABVD was observed in pts with sCD30 >median with a 2-yr mPFS of $68.9 \%$ [sCD30 >median] and $85.7 \%$ [sCD30 smedian]). A mPFS benefit in 
favor of $A+A V D$ vs $A B V D$ was observed in pts with $\mathrm{SCD} 30>$ median $(\mathrm{HR}(95 \% \mathrm{Cl})=0.600(0.428-0.841))$. Multivariate Cox analysis with the interaction between treatment group and SCD30 level showed an increased risk of experiencing an mPFS event with ABVD and sCD30 $>$ median (interaction $p=0.025$ ) when adjusted by other prognostic factors (Ann Arbor stage, IPS and ENI). Similar trends were observed with the exploratory ad-hoc TARC analysis. No new safety signals were reported in subgroups with elevated SCD30 or TARC levels.

Conclusions: Preliminary adhoc analysis indicates that ABVD treated patients do not perform as well with elevated baseline sCD30 and TARC levels. A+AVD treated patients perform well regardless of levels of these poor prognostic markers. Prospective studies need to be conducted in order to further validate these findings. If validated, these biomarkers may help identify patient populations that could benefit from more effectively targeted therapy.

Keywords: ABVD; brentuximab vedotin; classical Hodgkin lymphoma (cHL).

Disclosures: Radford, J: Consultant Advisory Role: Millennium Pharmaceuticals Inc, ADC Therapeutics, BMS, Novartis; Stock Ownership: GSK, AstraZeneca (spouse); Honoraria: Millennium Pharmaceuticals Inc, Seattle Genetics, Novartis, BMS; Research Funding: Millennium Pharmaceuticals, Inc., a wholly owned subsidiary of Takeda Pharmaceutical Company Limited. Connors, J: Consultant Advisory Role: Seattle Genetics, Millennium Pharmaceuticals Inc; Honoraria: Seattle Genetics, Millennium Pharmaceuticals Inc; Research Funding: Seattle Genetics. Younes, A: Consultant Advisory Role: BMS, Incyte, Janssen, Genentech, Merck; Honoraria: Genentech, Merck, Millennium Pharmaceuticals Inc, Incyte, BMS, AbbVie; Research Funding: Novartis, J\&J, Curis, Roche, BMS. Ansell, S: Research Funding: BMS, Seattle Genetics, Trillium, Affimed, Pfizer, LAM Therapeutics, Millennium Pharmaceuticals, Inc., a wholly owned subsidiary of Takeda Pharmaceutical Company Limited. Kim, W: Research Funding: Roche, Millennium Pharmaceuticals, Inc., a wholly owned subsidiary of Takeda Pharmaceutical Company Limited, $J \& J$, Mundupharma, Kyowa-kirin, Celltrion, DongaN. Flinn, I: Consultant Advisory Role: Abbvie, Seattle Genetics, TG Therapeutics, Verastem; Research Funding: Abbvie, Acerta Pharma, Agios, ArQule, AstraZeneca, BeiGene, Calithera Biosciences, Celgene, Constellation Pharmaceuticals, Curis, FORMA Therapeutics, Forty Seven, Genentech, Gilead Sciences, Incyte, Infinity Pharmaceuticals, Janssen, Juno Therapeutics, Karyopharm Therapeutics, Kite Pharma, Merck, MorphoSys AG, Novartis, Pfizer, Pharmacyclics, Portola Pharmaceuticals, Roche, Millennium Pharmaceuticals, Inc., a wholly owned subsidiary of Takeda Pharmaceutical Company Limited, Teva, TG Therapeutics, Trillium Therapeutics, Unum Therapeutics, Verastem. Pettengell, R: Consultant Advisory Role: CTI Life Sciences Ltd, Immune Design, Pfizer, Roche Ltd, Servier, Millennium Pharmaceuticals Inc, TEVA; Honoraria: CTI Life Sciences Ltd, Immune Design, Pfizer, Roche Ltd, Servier, Millennium Pharmaceuticals Inc, TEVA. Onsum, M: Employment Leadership Position: Seattle Genetics; Stock Ownership: Seattle Genetics. Josephson, N: Employment Leadership Position: Seattle Genetics, Inc.; Stock Ownership: Seattle Genetics, Inc.. Kuroda, S: Employment Leadership Position: Takeda Pharmaceutical Company Limited. Liu, R: Employment Leadership Position: Millennium
Pharmaceuticals, Inc., a wholly owned subsidiary of Takeda Pharmaceutical Company Limited. Miao, H: Employment Leadership Position: Millennium Pharmaceuticals, Inc., a wholly owned subsidiary of Takeda Pharmaceutical Company Limited. Gautam, A: Employment Leadership Position: Millennium Pharmaceuticals, Inc., a wholly owned subsidiary of Takeda Pharmaceutical Company Limited; Stock Ownership: Takeda Pharmaceutical Company Limited. Trepicchio, W: Employment Leadership Position: Millennium Pharmaceuticals, Inc., a wholly owned subsidiary of Takeda Pharmaceutical Company Limited. Sureda, A: Consultant Advisory Role: Millennium Pharmaceuticals Inc, BMS, Gilead, Novartis; Honoraria: Millennium Pharmaceuticals Inc, BMS, MSD, Gilead, Novartis, Jannssen, Celgene, Sanofi, Roche.

\section{6}

DOSE DENSE ABVD (DD-ABVD) AS FIRST LINE THERAPY IN EARLY-STAGE UNFAVORABLE HODGKIN LYMPHOMA (HD): RESULTS OF A PHASE II, PROSPECTIVE STUDY BY FONDAZIONE ITALIANA LINFOMI

R.Mazza $^{1}$ | M. Spina ${ }^{2}$ | C. Califano ${ }^{3}$ | F. Gaudio ${ }^{4}$

M. Carella $^{5}$ | U. Consoli ${ }^{6}$ | F. Palombi ${ }^{7}$ |

M. Musso $^{8}$ | A. Pulsoni ${ }^{9}$ | S. Kovalchuk ${ }^{10}$ |

M. Bonfichi ${ }^{11}$ | F. Ricci ${ }^{1}$ | A. Fabbri' ${ }^{12}$ |

A. Liberati ${ }^{13}$ | M. Rodari ${ }^{14}$ | L. Giordano ${ }^{15}$ |

M. Balzarotti ${ }^{1}$ | A. Gallamini ${ }^{16}$ | U. Ricardi ${ }^{17}$ |

S. Chauvie $^{18}$ | F. Merli ${ }^{19}$ | C. Carlo-Stella ${ }^{20}$ |

A. Santoro ${ }^{20}$

${ }^{1}$ Medical Oncology and Hematology Department, Humanitas Research Hospital, Rozzano-Milano, Italy; ${ }^{2}$ Medical Oncology Division, Centro Riferimento Oncologico, Aviano, Italy; ${ }^{3}$ Onco-Hematology Department, A. Tortora Hospital, Pagani, Italy; ${ }^{4}$ Emergency and Transplantation Department, Hematology section, University of Bari, Bari, Italy; ${ }^{5}$ Hematological Consultant, Casa di Cura La Madonnina, Milano, Italy; ${ }^{6}$ Garibaldi Nesima Hospital, Hematology Department, Catania, Italy; ${ }^{7}$ Hematology Department, Regina Elena National Cancer Institute, Roma, Italy; ${ }^{~}$ Onco-Hematology Unit, Casa di Cura "La Maddalena", Palermo, Italy; ${ }^{9}$ Cellular Biotechnologies and Hematology Department, Policlinico Umberto I, Sapienza University, Rome, Italy; ${ }^{10}$ Hematology Department, AOU Carreggi, Firenze, Italy; ${ }^{11}$ Hematology Division, IRCCS Policlinico S. Matteo, Pavia, Italy; ${ }^{12}$ Hematology Unit, AOU Senese, Siena, Italy; ${ }^{13}$ Onco-Hematology Division, S. Maria Hospital, Terni, Italy; ${ }^{14}$ Humanitas Reserch Hospital, Nuclear Medicin Unit, Rozzano-Milano, Italy; ${ }^{15}$ Biostatistic Unit, Humanitas Research Hospital, Rozzano, Milano, Italy; ${ }^{16}$ Research, Innovation and Statistc Depatment, Centre Antoine-Lacassagne, Nice, France; ${ }^{17}$ Oncology Department, Radiation Oncology, University of Torino, Torino, Italy; ${ }^{18}$ Medical Physics Department, S Croce e Carle Hospital, Cuneo, Italy; ${ }^{19}$ Hematology Department, Arcispedale Santa Maria Nuova, Reggio Emilia, Italy; 